\title{
Robust Optimized Discrete Wavelet Transform-Singular Value Decomposition Based Video Watermarking
}

\author{
Swaraja Kuraparthi ${ }^{*}$, Meenakshi Kollati, Padmavathi Kora
}

GRIET, Hyderabad 500090, India

Corresponding Author Email: k.swaraja@griet.ac.in

https://doi.org/10.18280/ts.360612

Received: 2 September 2019

Accepted: 13 November 2019

\section{Keywords:}

$A B C, D W T$, imperceptibility, robustness, SVD transform

\begin{abstract}
The effortless editing, interchanging and replication of multimedia data on the internet is growing exponentially and has created copyright protection uncertainties for content providers. Thus, in order to discourage illegal duplication and to attain the required level of protection to digital data, digital watermarking is found to be a feasible solution. Thus, this paper proposes a video watermarking technique by exploring Discrete Wavelet Transform (DWT) and Singular Value Decomposition (SVD) transform in addition to Artificial Bee colony (ABC) Optimization algorithm. In this paper, DWT is applied on every luminance frame which is divided into $8 \times 8$ blocks of the video ' $\mathrm{V}$ ' thus producing distinct frequency sub-bands. Out of them, LL band is selected for watermark insertion. Later SVD transform is implemented on the selected dwt blocks of LL bands of all frames. The starting indices of best blocks are obtained adaptively rather than manually through $\mathrm{ABC}$ algorithm. At the receiving part, retrieval of watermark contents is achieved by a similar evaluation scheme practiced during the embedding procedure. The proposed optimized DWT-SVD based video watermarking method has been evaluated in the presence of video processing attacks and simulation results proved that due to cascading of two powerful mathematical transforms DWT and SVD in addition to $\mathrm{ABC}$ algorithm the proposed video watermarking method endures all attacks and aptly extracts the concealed watermark without significant degradation in the video quality of the watermarked video. Thus when the Peak Signal to Noise Ratio (PSNR) and Normalized Correlation (NC) performance of the proposed algorithm is correlated with other related techniques it is found that the PSNR of the proposed method is above $53 \mathrm{~dB}$ for all set of videos and Robustness of the scheme is superior than the existing schemes for similar set of videos in terms of NC.
\end{abstract}

\section{INTRODUCTION}

With the increase in large digital data transfers on the Internet and the widespread usage of digital media has generated a lot of curiosity towards safeguarding intellectual property rights of multimedia document owners to protect their artifacts from misuse. Therefore, Safety and copyright fortification are becoming imperative issues in multimedia applications. Watermarking digital image/video is a procedure utilized for copyright security of digital media. Watermark is a data related to owner concealed into the digital media without disturbing its perceptual quality. In the event of any disagreement, the watermark content can be identified or extracted, moreover utilized as an evidence of ownership. The techniques in watermarking that do not entail the original image for extraction of the watermark are called "blind" watermarking. Digital watermarking technology mainly used in applications [1] such as Copyright fortification, continuous checking, Validation, Security and Indiscernible Communications. Digital watermarking persists to survive even after acquiring the data at the receiver side. If subtle data is disclosed to illicit personal, the digital watermark can be utilized to trace back to the intended recipient. Digital watermarking is used in concealed communication as an additional level of protection in contrast to cryptography. Though, the assailant is conscious of the endurance of the secret key, can be confident that within adequate interval he can decode the information, whereas while inserting the watermark digitally, the assailant can in no way be assured that hidden content is being conveyed. The foremost benefit of digital watermarks is that it lasts to persist though the receiver acquires the material. In applications related to medical [2, 3], watermarks are accomplished for credentials and retrieval of distinct patient archives. This specific application may avert human faults such as record discrepancy consequently precluding mortal faults.

The watermark in the proposed method is ingrained in the video clip frames. Methodologies in video watermarking can be ordered into two leading classes based on the process of concealing watermark data in the original video. The two classes are: Spatial domain watermarking as well as transformdomain watermarking [4-6]. In spatial-watermarking procedures, embedding and recognition are accomplished based on values of spatial pixels of the entire video frame. Spatial-domain schemes are simple to execute; on the contrary they are not resilient in conflict to accustomed digital signal processing functions such as video coding. Watermarking in the transform domain is more protected and stronger. Transform-domain schemes, in contrast, amend spatial pixel values of the host video in relation to encoded transform. Recurrently exercised transforms are the Discrete Cosine Transform (DCT) [7, 8], the Fast Fourier Transform (FFT), the 
Redundant Discrete Wavelet Transform (RDWT) [9], Discrete Wavelet Transform (DWT), Curvelet transform [10] and the Singular Value Decomposition (SVD) [11]. Transformdomain schemes of watermarking are established to be stronger besides unnoticeable when assessed in contradiction to spatial domain methods, subsequently dispersing the watermark in the spatial domain of video frame, makes it very tough to eliminate the inserted watermark. Every transform has its specific characteristics and indicates the image in diverse ways. SVD and Schur decompositions are two mathematical tools used to analyze matrices. When some perturbation occurs in the watermarked image, the extraction of the watermark is not affected much.

The watermark is concealed in the uppermost two levels of a 4-level Dual Tree Complex Wavelet Transform (DT-CWT) decomposition of the luminance (Y) channel [12]. They employed DT-CWT which inherits important properties of both the transforms DWT and Continuous Wavelet Transform (CWT) such as perfect reconstruction, better directional selectivity and approximate shift invariance. The authors embedded watermark in third and fourth decomposition levels of DT-CWT. The number of coefficients in first level decomposition is same as the number of coefficients in fourth level frame decomposition. This assures the data may be concealed many times and may be placed at different places. The watermarking scheme withstand attacks such as H.264 compression, small rotation angles, cropping with an average PSNR of $41 \mathrm{db}$. A video watermarking scheme is developed in compressed domain [13] for high quality High Definition (HD) videos for broadcasting services. The algorithm implemented in blind manner based on quantization index modulation which does not require cover video at the receiving end. The algorithm withstands attacks such as transcoding, frame rate changes and downscaling. However, the authors also emphasised that it is poor to robustness to geomentric attacks such as rotation. The watermark is concealed in the minimal sequences [14], by modulating the low-frequency coefficients of DCT. Though this technique in resolution is robust to downscaling, it is still unacceptable in terms of rotation and cropping. Wavelet based blind watermarking scheme is proposed for video sequences [15]. In this scheme, a dissimilar dimension block is concealed randomly in such selected blocks. A scene based video watermarking using Wavelet transform is proposed for concealing the watermark [16]. A wavelet transform is practiced while concealing the watermark in the video. A new video watermarking scheme is presented in which the watermark is concealed in the frames which has more intensity, texture and motion [17]. This process aid in enhancing the robustness of the video watermarking. A video watermarking scheme for concealing different portions of the watermark in dissimilar scenes of video is developed [18]. This method was so robust and can survive to several attacks like frame averaging, frame dropping, etc., but the higher time complexity was the foremost limitation. The watermark is embedded in selected coefficients in $8 \times 8$ blocks of slant transform [19] based on Human Visual System (HVS). The algorithm is found to be robust to scaling, rotation, frame swapping and frame averaging.

None of the existing watermarking schemes withstand to all types of chosen attacks because in the watermarking, there is a trade-off between the Robustness and Imperceptibility. If robustness of the scheme is improved, degradation in the video is scene. If imperceptibility is preserved it is not robust to attacks such as rotation, cropping and transcoding. Thus, nature inspired optimization algorithms [20, 21] have appeared as powerful tools to optimize parameters in many image processing applications. In the proposed work we also exploited the $\mathrm{ABC}$ method which is an optimization scheme to obtain improved results concerned to Imperceptibility and robustness.

The main advantage of the proposed algorithm is that it offers superior performance in terms of Robustness and Imperceptibility compared to reported techniques proposed by other authors, due to executing hybrid transforms (DWT and SVD) along with $\mathrm{ABC}$ optimization algorithm. Concern to DWT, the primary advantages of wavelet transform for watermarking applications include space spectrum localization, multi-resolution illustration, multi-scale examination, adaptability and linear intricacy. Watermarking with wavelets is also well-suited with the JPEG 2000 image standard. Because of its outstanding spatio-spectrum localization properties, the DWT is appropriate to determine the concealing parts in the host video where a watermark can be imperceptibly concealed. The mathematical properties of SVD is very attractive where even slight discrepancies of singular values do not disturb the visual perception of the host video, which inspires the concealing procedure of watermark to attain better performance in terms of transparency and robustness in contrast to DWT and SVD when executed separately. The main disadvantage of watermarking in pixel or transform domain is that the two watermarking performance constraints Transparency and Robustness are mutually tradeoff to each other. Thus, in recent years to optimize transparency and Robustness concurrently transform domain methods are incorporated with optimization methods such as Fuzzy Logic [22], ABC Algorithm [23-27], Genetic Algorithm (GA) [28], Particle Swarm Optimization (PSO) [29] and Particle Swarm Bacterial Foraging Optimization algorithm (PBSFO) [2]. Therefore, in the proposed work ABC optimization algorithm is implemented to optimize both Transparency and Robustness simultaneously to offer guaranteed authenticity and security of transmitted video information. Also, when the performance of the proposed method concern to PSNR and NC is contrasted with other reported practices, it is found that the proposed method offers superior performance due to implementation of hybrid optimized video watermarking algorithm.

In this work several major contributions offered to the watermarking field are: utilization of hybrid watermarking techniques (DWT and SVD) instead of single transform to exploit the attractive mathematical properties of both transforms and thus the performance is improved mainly in terms of Transparency and Robustness rather than applying DWT and SVD independently. From the discussions of the literature it may be noted that DWT provides time-spectrum localization, frequency at multi-resolution while adaptive selection of coordinate points selected for best DWT blocks provide Security, Robustness and visual Imperceptibility performance. $\mathrm{ABC}$ algorithm due to its simplicity, ease of implementation and also usage of fewer control parameters has captured attention of authors to use it in the proposed algorithm. In ABC algorithm, to reach global optima, a robust search space is carried by both exploration and exploitation process. The exploitation process in $\mathrm{ABC}$ is carried by onlooker and employee bees, further the exploration process is carried by scout bees. In this work, instead of manually selecting the coordinates of best DWT blocks, optimized 
coordinate points obtained from $\mathrm{ABC}$ are used. $\mathrm{ABC}$ has been already implemented for resolving complex optimization problems amazingly in various fields and is now applied for enhancing the quality and robustness of video watermarking scheme for the first time in this work to the best of authors knowledge.

This paper is systematized as follows. Different methodologies such as DWT and SVD transform along with $\mathrm{ABC}$ algorithm is deliberated in section 2. Proposed algorithm is presented in section 3. Simulation outcomes are presented and compared with the existing methods in section 4 . Conclusions are specified in section 5.

\section{METHODOLOGIES}

The disparate theories and practices exploited in this work are deliberated in this section so as to acquaint the reader with the elementary concepts.

\subsection{Singular value decomposition (SVD)}

A symmetric matrix is fragmented by SVD into three submatrices in which singular values are alienated into diagonal matrix [8, 11]. This progression is also recognized as diagonalization of matrix. The three matrices that have undergone decomposition are termed as left singular matrix (L), singular matrix (W) and right singular matrix (R). Assuming $\mathrm{C}$ as a symmetric matrix SVD can be calculated with Eq. (1).

$$
C=L \boldsymbol{W} R^{T}
$$

In Eq. (1), Matrix $\mathbf{W}$ appear as a diagonal matrix which is rectangular. The magnitude of diagonal values of matrix $\mathbf{W}$ are organized in a descendant order. Such diagonal values are identified as singular values of matrix $\mathrm{C}$. For matrix $\mathrm{C}$ with $\mathrm{m} \times \mathrm{m}$ order, Singular matrix $(\mathrm{W})$ can comprise of utmost $\mathrm{m}$ diagonal values. Layer wise involvement of $\mathrm{L}$ and $\mathrm{R}$ matrices in the matrix $\mathrm{C}$ is represented through singular values; over which the fragmentation is accomplished. The matrix quality becomes worse when the order of regeneration of matrix decreases. In contrast, there is no much disturbance in the quality of matrix with a minor disparity in the singular values. Eq. (2) shows one of the most important properties of singular values of diagonal matrix $\mathrm{W}$.

$$
f_{1} \geq f_{2} \geq f_{3} \geq \cdots>f_{k+1}>f_{k+2}=f_{m}
$$

where, ' $\mathrm{k}$ ', $(\mathrm{k} \leq \mathrm{m})$ indicates the rank concerned to singular matrix $\mathrm{W}, \mathrm{f}_{1}, \mathrm{f}_{2}, \mathrm{f}_{3}, \ldots \mathrm{f}_{\mathrm{m}}$ symbolizes the diagonal values and $\mathrm{m}$ denotes the order of matrix. The matrices $\mathrm{L}$ and $\mathrm{R}$ likewise follow $L L^{T}=I_{m}$ and $R R^{T}=I_{m}$.

\subsection{Discrete wavelet transform (DWT)}

The knowledge of wavelets is exploited by DWT to depict the images in an energy compact manner $[2,3]$. Wavelets are delineated as the small waves with frequencies which are always changing. DWT offers a great energy packaging capacity and explicitly it is the reason to apply in numerous image processing needs such as compression of the image, watermarking of the image, etc. In DWT, the novel image is changed into wavelet domain, consisting of four bands.
Among the four sub-bands, three (HL, LH and $\mathrm{HH}$ ) are identified as sub-images which encompass high frequencies with fringe data. The other sub-band (LL) comprises of low frequency, termed as approximate sub-image since it holds an estimated data of original image. It is attained by low-pass filtering together in the row as well as column directions. Additionally, decomposition of sub-bands is feasible to reach higher level of decomposition. This progression can be passed to numerous levels as required in the application. When related to HL, LH and HH sub-bands, LL sub-band proves to be more stable in nature and it is extremely tough to get transformed by attacks as this band holds principal portion of energy of original image. This feature makes the approximate sub-band more apt for concealing the watermark with robustness. The exceptional spatio-frequency localization property pertained to DWT is exploited by disparate image watermarking methods in recent to contribute a raised value with regard to imperceptibility besides robustness. Figure 1 depicts disparate levels concerned to discrete wavelet transform.

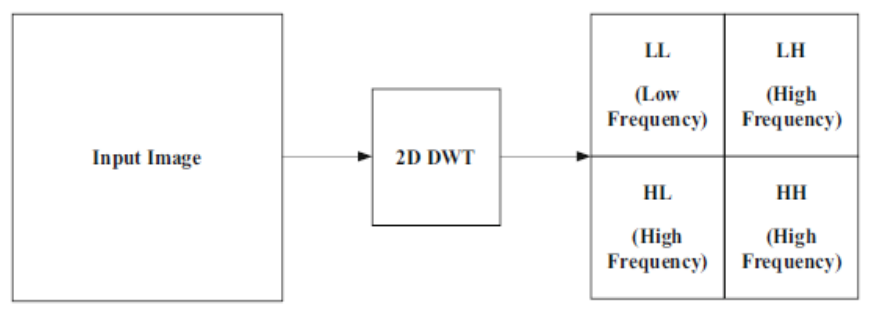

Figure 1. First level decomposition of wavelet for an image

\subsection{Artificial bee colony (ABC)}

Among several evolutionary procedures [23] identified Artificial Bee Colony (ABC) as one such simple, rapid population based method and performance wise also it is too robust. $\mathrm{ABC}$ is practiced effectively on disparate domains to offer relatively raise in results [24-27]. Analogous to other Evolutionary procedures, variable values which are optimal are to be inspected by exploiting the $\mathrm{ABC}$ method with the aim of maximizing/ minimizing the specified cost function. Simulation outcomes demonstrates that ABC attains identical results comparable to several dissimilar metaheuristics practices along with this it also presents an extra benefit of adopting fewer control constraints. $\mathrm{ABC}$ is familiar for its capability to explore the search related to multidimensional which is the main focus of the proposed work also. The basic steps intended for implementation are realized from $\mathrm{ABC}$ algorithm [27].

$\mathrm{ABC}$ method functions according to the smart foraging behavior of the honey bee swarms. The feasible solutions for any specified issue are categorized by the food source in ABC scheme, further the fitness of any outcome is measured by the quantity of nectar in a food source. Three forms of bees arise in $\mathrm{ABC}$, termed as working bees, spectator bees and detective bees. Working bees directs the number of explanations in the specified population dimensions. $\mathrm{ABC}$ is originated with an initial population of results of dimension L (places where food is available) with each having a dimension X. i.e., early solution can be symbolized as $\mathrm{P}_{\mathrm{k}}=\{\mathrm{p}(\mathrm{k}, 1), \mathrm{p}(\mathrm{k}, 2) \ldots \mathrm{p}(\mathrm{k}, \mathrm{X})\}$; Where $\mathrm{k}=1,2 \ldots \mathrm{L}$.

The searching process is a repetitive procedure subsequent to the initial circulation, while selecting optimum solution until the ending state is obtained. The spectator bees opt for the sources of food which are finest, depending on the value 
of fitness function in addition to the information provided by working bees, while the detective bees kept their recent sources of food with the purpose of exploring improved sources of food. In $\mathrm{ABC}$ procedure, working bees and spectator bees are answerable for the process of exploitation, while detective bees take attention of appropriate inspection.

The $\mathrm{ABC}$ algorithm encompass the following stages:

1. The sources of food with $\mathrm{L}$ number are preferred arbitrarily among the acceptable lower $\mathrm{P}_{\min }=\left(\mathrm{p}_{(\min , 1), \ldots}\right.$ $\left.\mathrm{p}_{(\min , X)}\right)$ as well as upper limit $\mathrm{P}_{\max }=\left(\mathrm{p}_{(\min , 1), \ldots} \mathrm{p}_{(\min , X)}\right)$ of distribution with each including dimension of $\mathrm{X}$ through Eq. (3).

$$
p_{(k, l)=} p_{(\min , l)}+\operatorname{rand}(0,1) \times\left(p_{(\max , l)}-p_{(\min , l)}\right)
$$

2. All working bees produces a novel result with the local information accessible to it and relates the fitness of engendered result with the parent result. The improved result between these two is utilized for succeeding iteration. The novel result $\mathrm{Q}_{\mathrm{k}}$ is engendered from existing result by making use of Eq. (4)

$$
q_{(k, l)}=q_{(k, l)}+\Phi_{(k, l)} \times\left(q_{(k, l)}-p_{(m, l)}\right)
$$

Here $\Phi_{(k, l)}$ is an arbitrarily acquired numeral between [$1,1]$ and indices $m €(1,2, \ldots \mathrm{L})$ and $1 €(1,2, \ldots \mathrm{X})$ are arbitrarily acquired in such a form that $\mathrm{m}$ and 1 persist to be unlike.

3. The working bee distributes the fitness details with the spectator bee. The spectator bee produces a probability $(\mathrm{Pi})$ of nectar (fitness) quantity by means of Eq. (5) and Eq. (6).

$$
\begin{gathered}
F_{K}=\frac{f i t_{K}}{\sum_{k=1}^{L} f i t_{K}} \\
\text { fit }_{k}=\left\{\begin{array}{c}
\frac{1}{f\left(Y_{k}\right)+1}, \quad \text { iff } f\left(Y_{k}\right) \geq 0 \\
1+a b s\left[f\left(Y_{k}\right)\right], \text { otherwise }
\end{array}\right.
\end{gathered}
$$

At this point $f\left(Y_{k}\right)$ denotes the value of objective function at the position of food $Y_{k}$.

4. An arbitrary numeral is engendered for all spectator bees within zero and one; if $\mathrm{Fk}$, the position of food possesses larger value than the arbitrary numeral then step 2 is tracked by the spectator bee as well.

5. If prearranged number of repetitions cannot alter the positions of food, at that time such positions are supposed to be eliminated. The value of prearranged numeral of repetitions is considered as significant constraint and identified as boundary. In such circumstances, detective bees regulate the novel places arbitrarily with the aim of exchanging the places that are eliminated.

Stages 1 to 5 are reproduced up to the prearranged ending condition (maximum repetition, least variation) encounter.

\section{PROPOSED ABC BASED DWT-SVD VIDEO WATERMARKING TECHNIQUE}

\subsection{Watermark embedding}

The planned watermarking method comprises primarily of three phases; watermark concealing, watermark recovery and computing the best Sel_dwtblock. YUV QCIF videos are used. The frames are in true color represented by RGB color components. The luminance component is not affected much by amendments compared with chrominance components $\mathrm{Cb}$ and $\mathrm{Cr}$. Therefore, for implanting watermark, luminance components of frames are used rather than $\mathrm{Cb}$ and $\mathrm{Cr}$. The procedure of embedding and extracting the watermark is shown in Figures $2 \& 3$ respectively. To conceal owners logo or watermark in luminance of frames in video the following steps are used.

Step 1: The original video ' $\mathrm{V}$ ' is segregated as set of $\mathrm{M}$ number of frames, the watermark ' $\mathrm{W}_{\mathrm{m}}$ ' is concealed inside the video 'V'.

Step 2: In the video, each true colored frame is transformed into $\mathrm{YC}_{\mathrm{b}} \mathrm{C}_{\mathrm{r}}$ color model from $\mathrm{RGB}$ and later the luminance component $(\mathrm{Y})$ is isolated from Chrominance components $(\mathrm{Cb}$ and $\mathrm{Cr}$ ) for watermark insertion.

Step 3: Apply DWT on each frame to segregate the frame into four bands namely LL, LH, HL and $\mathrm{HH}$ band as given in Eq. (7). As watermark embedded in LL band is robust to signal and compressional attacks, LL band is chosen for embedding watermark and rest of bands are untouched.

Step 4: Later, LL band are alienated into four $8 \times 8$ nonoverlapping blocks.

$$
V_{D W T}=D W T(V)
$$

Step 5: ABC method is employed primarily to elect particular four 8x8 blocks signified as Sel_dwtblock to embed watermark in every frame for attaining imperceptibility.

Step 6: Later SVD transform is executed on the concatenated four $8 \times 8$ Sel_dwtblocks to obtain left singular vector $\mathrm{L}$ and singular matrix $\mathrm{W}$ as well as right singular vector $\mathrm{R}$. The eigen values are as diagonal elements of W. Majority of the strength is preserved in the eigen values.

Step 7: It decomposes concatenated four $8 \times 8$ Sel_dwtblocks into $L_{v b}, W_{v b}$, and $R_{v b}$ as depicted in Eq. (8).

$$
C_{v}=L_{v b} \times W_{v b} \times R_{v b^{T}}
$$

Step 8: Similarly, SVD transform is applied to the watermark to obtain $L_{b w a t}$ and $R_{b w a t}{ }^{T}$, as shown in Eq. (9).

$$
W a t=L_{b w a t} \times W_{b w a t} \times R_{b w a t}^{T}
$$

Step 9: We add Wbwat to the Wvb matrix to obtain $\mathrm{W}_{\text {vbnew }}$ as given in Eq. (10).

$$
\mathrm{W}_{\mathrm{vbnew}}=\mathrm{Wvb}+\beta \mathrm{Wbwat}
$$

The selection of the ' $\beta$ ' coefficient depends on the compromise strength or imperceptibility preferred by the user.

Step 10. Inverse SVD is applied to obtain modified selected blocks as given in Eq. (11).

$$
C_{\text {vnew }}=L_{v b} W_{\text {vbnew }} R_{b v^{T}}
$$

Step 11: Reposition the modified blocks in the LL band to obtain $L L_{n e w}$.

Step 12: Combine $L L_{n e w}$ With LH, HL, HH to obtain the 
$V_{\text {DWTnew }}$. Apply inverse DWT to obtain watermarked frames.

$$
R_{\text {wat }}=D W T^{-1}\left(V_{\text {DWTnew }}\right)
$$

Step 13: Concatenate all frames to obtain watermarked video.

\subsection{Watermark extraction}

The gradual progression for the extraction of watermark in detail is illustrated in the following steps:

Step 1: The steps 1-6 in watermark embedding algorithm defined in subsection 3.1 is applied on watermarked and cover video $R_{\text {wat }}$ and $\mathrm{V}$ to obtain singular values of watermarked and cover video for the chosen four $8 \times 8$ Sel dwtblocks by exploring $\mathrm{ABC}$ method and obtain $\mathrm{L}, \mathrm{W}$ and matrix $\mathrm{R}$ as follows:

$$
C_{v^{\prime}}=L_{b w a t^{\prime}} \times W_{b w a t^{\prime}} \times R_{b w a t^{T}}
$$

Step 2: Substract singular values of host from singular values of watermarked video to obtain singular values of watermark as given in Eq. (14).

$$
\text { Wbext }=\frac{\mathrm{W}_{\text {vbnew }}-\mathrm{Wvb}}{\beta}
$$
$(15)$.

Step 3: The watermark is rebuild using the following Eq.

$$
W E_{E X T}=L_{w a t} W_{w a t} R_{w a t}
$$

where, $\mathrm{WE}_{\mathrm{EXT}}$ is the extracted watermark.

\subsection{Computation of best Sel_dwt blocks using ABC algorithm}

Karaboga [23] formulated an optimization algorithm based on small foraging action of a honey bee swarms known as Artificial Bee Colony (ABC) algorithm. A population of solutions is used in $\mathrm{ABC}$ algorithm instead of single solution. The population of solutions is represented by food sources in ABC algorithm. The solutions in the initial population are represented by employee bees. The algorithm initializes with a population of size $\mathrm{m}$ (food source locations), each has dimension d, i.e., initial solutions can be represented as $X_{j}=$ $\{x(j, 1), x(j, 2) \ldots x(j, d)\}$; where, $j=1,2, \ldots . m$ and $m=$ number of employee bees. The algorithm terminates when the number of generations is reached or converges or there is no change in the fitness function.

The four starting coordinates of chosen Sel_DWT blocks are utilized to apply in fitness function. These are randomly generated by ABC initially. These random starting coordinates are later fed to fitness function. The fitness function is computed using Eq. (16).

fitness function $^{=}$

$$
\frac{1}{\operatorname{Avg}\left(\operatorname{SSIM}\left(V_{\text {Frames }}, R_{\text {img Frames }}\right)\right)-\frac{1}{n} \sum_{i=1}^{n} N C C\left(W_{m}, W E_{E X T}\right)}
$$
by

The cost function is seeking for minimization and is given

$$
f_{i}=\frac{1}{\text { fitness_function }}
$$

In this equation $V_{\text {Frames }}$ and $R_{\text {img Frames }}$ are frames of host and watermarked video. SSIM is the structural similarity measure, given in $[\mathrm{km}]$. The SSIM values lies between 0 and 1.

After video subjected to signal processing and compression attacks, average NCC of the 9 attacks is taken. The average NCC tries to reach towards 1. So the cost function is minimized. The best coordinates where the cost function is low, they are considered for watermark concealing and extraction. The number of variables used in $\mathrm{ABC}$ are $\mathrm{X}, \mathrm{Y}$ coordinates of 4 blocks yielding 8 . The number of generations used is 100. The control parameter values used to execute ABC optimization algorithm is shown in Table 1. The computational speed of the proposed algorithm is 55.44 minutes.

Table 1. Control parameters used in ABC optimization algorithm

\begin{tabular}{cc}
\hline \multicolumn{2}{c}{ ABC Control Parameters } \\
\hline Number of Swarms & 20 \\
No,of generations & 100 \\
No.of onlooker bees & $40 \%$ of swarm \\
Number of employee bees & $60 \%$ of swarm \\
Number of scout bees & changeable \\
\hline
\end{tabular}

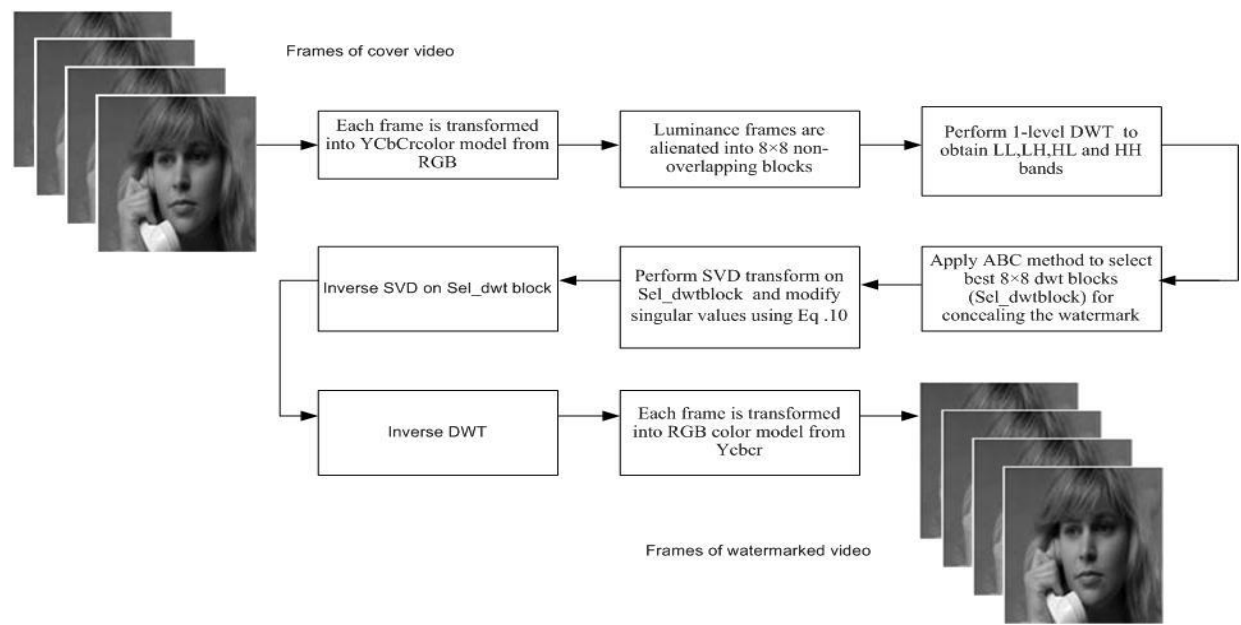

Figure 2. Procedure of embedding the watermark 

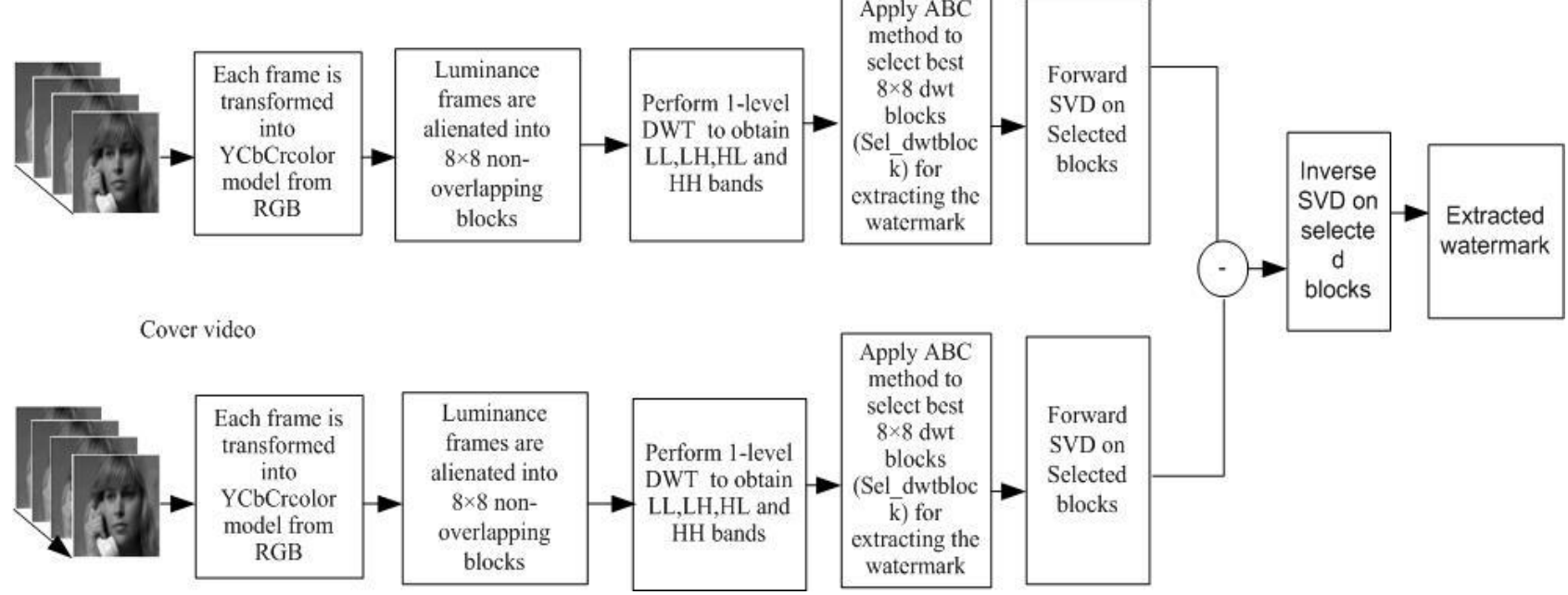

Figure 3. Procedure of Extracting the watermark

\section{SIMULATION RESULTS AND ANALYSIS}

The entire simulation results are assessed by finding the imperceptibility and robustness. The simulation results of the proposed algorithm are contrasted with existing algorithms $[13,14]$ for testing primarily two criteria's: Imperceptibility test as well as Robustness test. The superiority is shown in two evaluations; they are: Peak Signal to Noise Ratio (PSNR) on top of Normalized Correlation (NC) for mutually before and after attacks.

Table 2. Comparison in terms of PSNR of proposed algorithm with existing algorithms after different attacks on Suzie video

\begin{tabular}{cccc}
\hline \multirow{2}{*}{$\begin{array}{c}\text { Different types of } \\
\text { Attacks }\end{array}$} & \multicolumn{3}{c}{ PSNR } \\
\cline { 2 - 4 } & $\begin{array}{c}\text { Proposed } \\
\text { method }\end{array}$ & {$[\mathbf{1 4}]$} & {$[\mathbf{1 3}]$} \\
\hline (a) No attack & 59.93 & 48.12 & 58.45 \\
(b) Resizing & 54.22 & 48.98 & 52.34 \\
(c) Rotation & 57.63 & 49.54 & 54.67 \\
(d) Cropping & 53.58 & 39.99 & 50.78 \\
(e)Frame swapping & 54.44 & 47.34 & 51.16 \\
(f) Sharpening & 56.32 & 38.67 & 50.72 \\
(g)Frame Averaging & 54.45 & 44.54 & 53.69 \\
(h)Salt \&Pepper noise & 54.89 & 47.86 & 49.25 \\
(i)Gaussian noise & 54.12 & 43.97 & 52.02 \\
(j)Histogram Equalization & 53.04 & 39.35 & 50.30 \\
\hline
\end{tabular}

Table 3. Comparison in terms of NC of proposed algorithm with existing algorithms after different attacks on Suzie video

\begin{tabular}{cccc}
\hline Different types of Attacks & Proposed scheme & {$[\mathbf{1 4}]$} & {$[\mathbf{1 3}]$} \\
\hline (a) No attack & 0.99 & 0.9 & 0.93 \\
(b) Resizing & 0.99 & 0.84 & 0.93 \\
(c) Rotation & 0.95 & 0.89 & 0.9 \\
(d) Cropping & 0.98 & 0.89 & 0.91 \\
(e)Frame swapping & 0.88 & 0.7 & 0.8 \\
(f) Sharpening & 0.93 & 0.76 & 0.87 \\
(g)Frame Averaging & 0.91 & 0.79 & 0.91 \\
(h)Salt \&Pepper noise & 0.99 & 0.87 & 0.92 \\
(i)Gaussian noise & 0.9 & 0.85 & 0.86 \\
(j)Histogram Equalization & 0.89 & 0.6 & 0.82 \\
\hline
\end{tabular}

\subsection{For testing imperceptibility}

The simulated value of $\mathrm{NC}$ is 0.99 . the extorted watermark is almost alike to the original and precise extraction is accomplished. While relating the planned scheme with [14] and [13] the proposed scheme recorded a PSNR up to $59.93 \mathrm{db}$ for Suzie video which is superior to the PSNR stated by [14] and [13]. Table 2 demonstrates the PSNR assessment of the proposed video watermarking procedure with earlier hybrid optimized video watermarking methods. The performance evaluations of the planned method with former hybrid optimized video watermarking schemes are shown in Table 3 for the Suzie video sequence in terms of the NC.

\subsection{For testing robustness}

Robustness is the capability of detector to extort the masked watermark even after altering the watermarked data. Owing to enormous volumes of data and intrinsic redundancy between frames, the video used for embedding the watermark is extremely susceptible to plagiarize assaults which include averaging of frames, rotation, tumbling of frames, sharpening etc. Frequently robustness is assessed by the endurance of watermark after disparate varieties of attacks, such as Resizing of frames, Rotation of frames, Cropping the frame, swapping the frame, Sharpening the frame, averaging the frame, effect of noise like Salt \& pepper besides Gaussian and applying Histogram equalization on the frames, etc. The retrieved watermark after applying different types of attacks as shown in Figure 5 on Suzie video is depicted in Figure 6.

In the proposed scheme the $\mathrm{NC}$ values are always above 0.85, revealing the developed quality of the extracted watermark. Thus, the recommended algorithm illustrates upright robustness though the attack is resilient. The object ensuing to the greater performance of the planned algorithm under numerous kinds of attacks is as a result of relating superior categories of transforms in union to optimization procedure, therefore limited numerals of coefficients interrupt on the excellence of the restored watermark; also gradually it authorizes us to exploit highest entrenching feature with enhancement in robustness. 


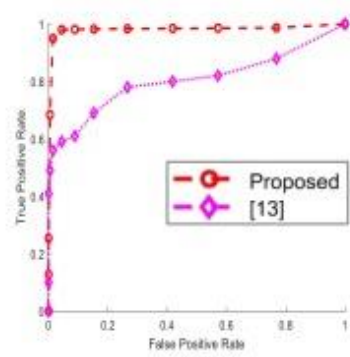

(a)

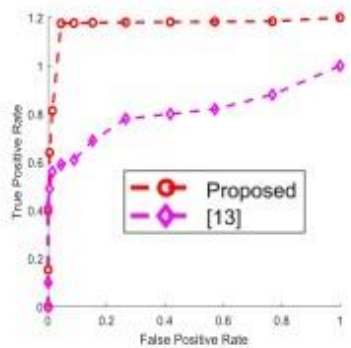

(d)

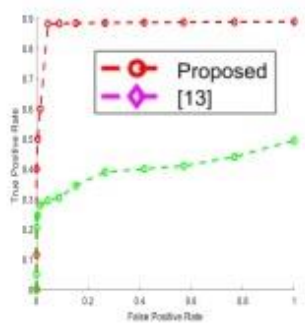

(g)

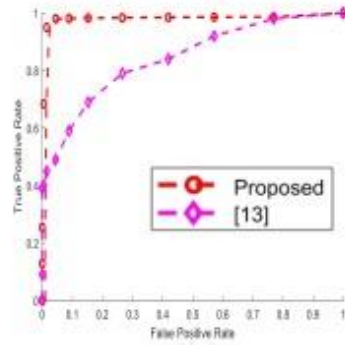

(b)

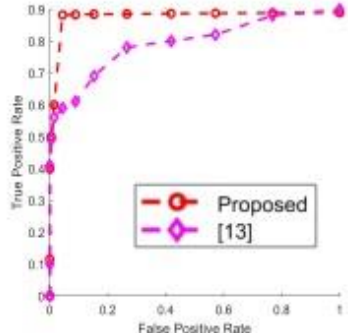

(e)

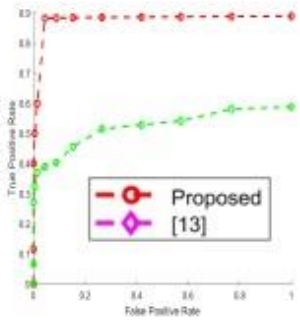

(h)

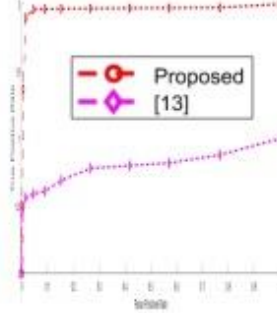

(c)

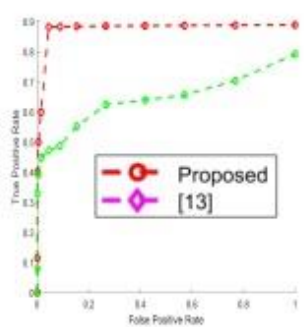

(f)

Figure 4. Receiver Operating Characteristic (ROC) curves for (a) Frame Resizing, (b) Frame Rotation, (c) Cropping the frame,

(d) swapping the frame, (e) Sharpening the frame, (f) Averaging the frame, (g) Salt \& pepper noise (h) Gaussian noise (i)

Histogram equalization
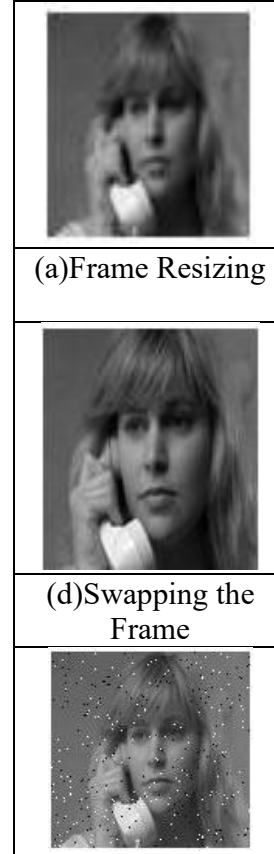

(g) Noise like Salt \&pepper

(h) Noise such as gaussian

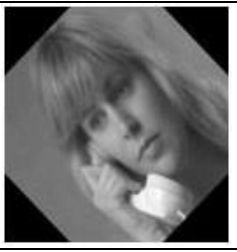

(b)Frame Rotation

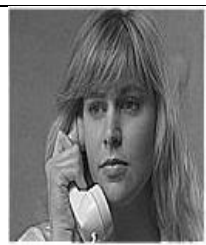

(e)Sharpening the Frame

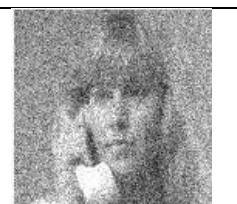
Frame

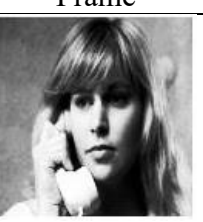

(i) Applying Histogram equalization on the Frame

Figure 5. Disparate varieties of attacks on the video of Suzie

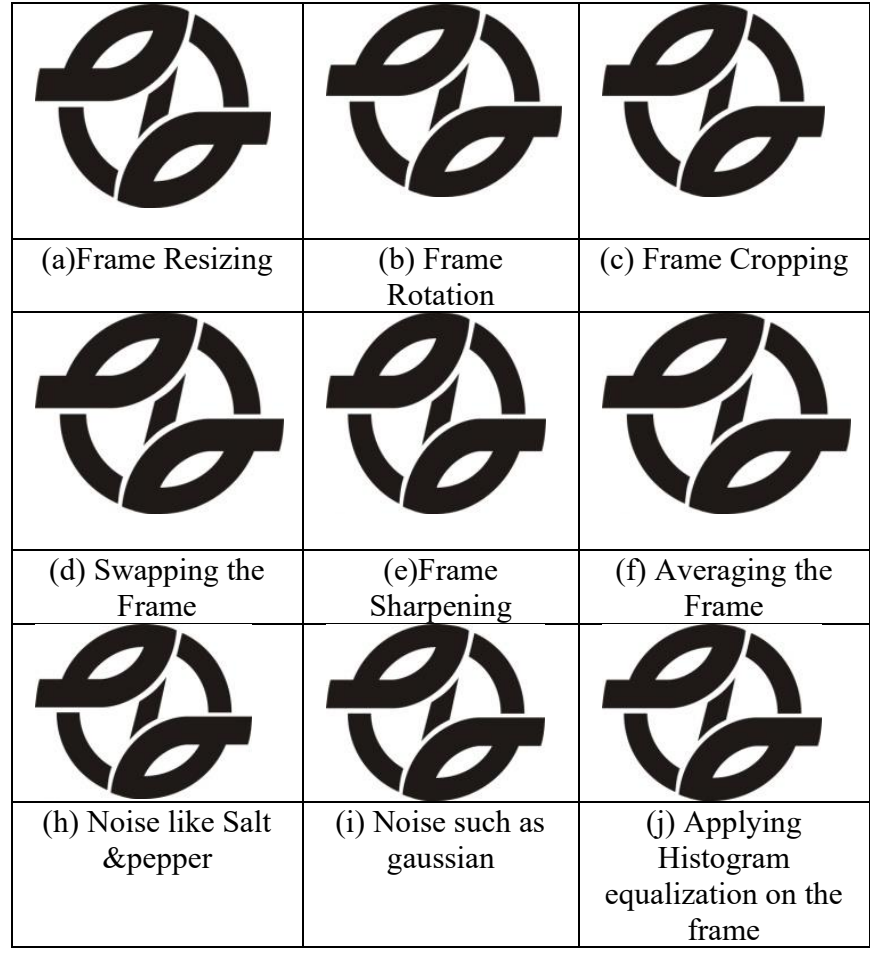

Figure 6. Extracted watermark after applying dissimilar types of attacks on the video of Suzie 


\subsection{Statistical analysis}

Statistical analysis based on Receiver Operating Characteristic (ROC) curves for different attacks on watermarked videos are performed in Figure 4 to further validate the proposed video watermarking algorithm. The proposed algorithm is compared with existing algorithm [13] in Figure 4 to show the statistical analysis by drawing the ROC curves for attacks such as Frame Resizing, Frame Rotation, Cropping the frame, Averaging the frame, Salt \& pepper noise, Gaussian noise and Histogram equalization. In watermarking, false alarm probability is the probability of recognizing the watermark, when the video is not watermarked and false rejection probability is the incapability to recover watermark when the video is watermarked. In this work, 30 video's of HD, Compressed Intermediate Format (CIF), Quarter Compressed Intermediate Format (QCIF) are used. ROC curves are drawn between the False positive rate and True positive rate at different thresholds at $99 \%$ confidence interval. The results attained by the ROC curves for the specified attacks from Figure 4 confirms that the proposed method has average detection probability of $99.6 \%$ compared to the paper [13] which means that $99.6 \%$ of video's are correctly authenticated in this work.

\section{CONCLUSIONS}

In the proposed video watermarking scheme, the coordinates of Best Dwt blocks were optimized with the help of ABC with optimized Transparency and Robustness. The watermark is inserted in best dwt blocks which again helped the scheme in achieving high robustness by preserving the video quality intact. In future, the video watermarking will be carried with $\mathrm{ABC}$ and other DWT variants and other Metaheuristic algorithm to improve the performance of the watermarking method.

\section{REFERENCES}

[1] Miller, M.L., Cox, I.J., Bloom, J.A. (2002). Digital Watermarking. Academic Press.

[2] Swaraja, K., Meenakshi, K., Kora, P. (2020). An optimized blind dual medical image watermarking framework for tamper localization and content authentication in secured telemedicine. Biomedical Signal Processing and Control, 55: 101665. https://doi.org/10.1016/j.bspc.2019.101665

[3] Swaraja, K. (2018). Medical image region based watermarking for secured telemedicine. Multimedia Tools and Applications, 77(21): 28249-28280. https://doi.org/10.1007/s11042-018-6020-7

[4] Swaraja, K., Latha, Y.M., Reddy, V.S.K., Paramkusam, A.V. (2011). Video watermarking based on motion vectors of H. 264. In India Conference (INDICON). Annual IEEE, Hyderabad, India, pp. 1-4. https://doi.org/10.1109/INDCON.2011.6139472

[5] Swaraja, K., Madhaveelatha, Y., Reddy, V.S.K. (2014). A pristine digital video watermarking in H. 264 compressed domain. In Computational Intelligence and Computing Research (ICCIC). IEEE International Conference, Coimbatore, India, pp. 1-4. https://doi.org/10.1109/ICCIC.2014.7238503
[6] Meenakshi, K., Prasad, K.S., Rao, C.S. (2017). Development of low complexity video watermarking with conjugate symmetric sequency - complex Hadamard transform. IEEE Communications Letters, 21(8): 1779-1782. https://doi.org/10.1109/LCOMM.2017.2700461

[7] Swaraja, K., Madhaveelatha, Y., Reddy, V.S.K. (2015). A secure method of optimized low complexity video watermarking. ARPN Journal of Engineering and Applied Sciences, 10(4):1822-1827.

[8] Meenakshi, K., Swaraja, K., Kora, P. (2019). A robust DCT-SVD based video watermarking using zigzag scanning. Soft Computing and Signal Processing, Springer, Singapore, pp. 477-485. https://doi.org/10.1007/978-981-13-3600-3_45

[9] Yasasvy, T., Sushil, K.V., Meenakshi, K., Swaraja, K., Kora, P. (2019). A hybrid blind watermarking with redundant discrete wavelet and Hadamard transform. International Journal of Innovative Technology and Exploring Engineering, 8(11): 2216-2220. https://doi.org/10.35940/ijitee.K2045.0981119

[10] Meenakshi, K., Kora, P. (2019). Video watermarking with curvelet transform. International Journal of Innovative Technology and Exploring Engineering (IJITEE), 8: 602-607.

[11] Meenakshi, K., Rao, C.S., Prasad, K.S. (2014). A robust watermarking scheme based Walsh-Hadamard transform and SVD using ZIG ZAG scanning. 2014 International Conference on Information Technology, Bhubaneswar, India, pp. 167-172. https://doi.org/10.1109/ICIT.2014.53

[12] Coria, L.E., Pickering, M.R., Nasiopoulos, P., Ward, R.K. (2008). A video watermarking scheme based on the dualtree complex wavelet transform. IEEE Transactions on Information Forensics and Security, 3(3): 466-474. https://doi.org/10.1109/TIFS.2008.927421

[13] Lee, M.J., Im, D.H., Lee, H.Y., Kim, K.S., Lee, H.K. (2012). Realtime video watermarking system on the compressed domain for high definition video contents: Practical issues. Digital Signal Processing, 22(1): 190198. https://doi.org/10.1016/j.dsp.2011.08.001

[14] Ling, H., Wang, L., Zou, F. (2011). Real-time video watermarking scheme resistant to geometric distortions. Journal of Electronic Imaging, 20(1): 013025. https://doi.org/10.1117/1.3565193

[15] Lin, W.H., Wang, Y.R., Horng, S.J., Kao, T.W., Pan, Y. (2009). A blind watermarking method using maximum wavelet coefficient quantization. Expert Systems with Applications, $\quad 36(9)$ : $\quad 11509-11516$. https://doi.org/10.1016/j.eswa.2009.03.060

[16] Masoumi, M., Amiri, S. (2013). A blind scene-based watermarking for video copyright protection. AEUInternational Journal of Electronics and Communications, 67(6): 528-535. https://doi.org/10.1016/j.aeue.2012.11.009

[17] Ko, C.C., Kuo, Y.L., Hsu, J.M., Yang, B.Z. (2013). A multi-resolution video watermarking scheme integrated with feature detection. Journal of the Chinese Institute of Engineers, $36(7)$ : 878-889. https://doi.org/10.1080/02533839.2012.747057

[18] Singh, T.R., Singh, K.M., Roy, S. (2013). Video watermarking scheme based on visual cryptography and scene change detection. AEU-International Journal of Electronics and Communications, 67(8): 645-651. https://doi.org/10.1016/j.aeue.2013.01.008 
[19] Meenakshi, K., Rao, S.C., Prasad, S.K. (2014). A scene based video watermarking using slant transform. IETE Journal of Research, 60(4): 276-287. https://doi.org/10.1080/03772063.2014.961570

[20] Ansari, I.A., Pant, M. (2015). SVD watermarking: Particle swarm optimization of scaling factors to increase the quality of watermark. In: Proceedings of Fourth International Conference on Soft Computing for Problem Solving. Springer India, pp. 205-214. https://doi.org/10.1007/978-81-322-2220-0_17

[21] Ansari, I.A., Pant, M., Ahn, C.W. (2016c). ABC optimized secured image watermarking scheme to find out the rightful ownership. Optik International Journal for Light and Electron Optics, 127(14): 5711-5721. https://doi.org/10.1016/j.ijleo.2016.03.070

[22] Meenakshi, K., Bethel, G.B. (2014). Design and simulation of constant bit rate compressor using fuzzy logic. In 2014 First International Conference on Networks \& Soft Computing (ICNSC2014), pp. 309-313. https://doi.org/10.1109/CNSC.2014.6906697

[23] Karaboga, D., Akay, B. (2009). A comparative study of Artificial Bee Colony algorithm. Applied Mathematics and Computation, 214(1): 108-132. https://doi.org/10.1016/j.amc.2009.03.090

[24] Draa, A., Bouaziz, A. (2014). An artificial bee colony algorithm for image contrast enhancement. Swarm and
Evolutionary Computation, 16: https://doi.org/10.1016/j.swevo.2014.01.003

69-84.

25] Akay, B. (2013). A study on particle swarm optimization and artificial bee colony algorithms for multilevel thresholding. Applied Soft Computing, 13(6): 3066-3091. https://doi.org/10.1016/j.asoc.2012.03.072

[26] Hanbay, K., Talu, M.F. (2014). Segmentation of SAR images using improved artificial bee colony algorithm and neutrosophic set. Applied Soft Computing, 21: 433443. https://doi.org/10.1016/j.asoc.2014.04.008

[27] Ansari, I.A., Pant, M., Ahn, C.W. (2016d). Artificial bee colony optimized robust-reversible image watermarking. Multimedia Tools and Applications, 76: 18001-18025. https://doi.org/10.1007/s11042-016-3680-z

[28] Aslantas, V. (2008). A singular-value decompositionbased image watermarking using genetic algorithm. AEU-International Journal of Electronics and Communications, 62(5): 386-394. https://doi.org/10.1016/j.aeue.2007.02.010

[29] Singh, P., Agarwal, S. (2013). A Hybrid DCT-SVD Based Robust watermarking scheme for copyright protection. International Conference on Emerging Trends in Engineering and Technology (ICETET'2013) Dec. 7-8, 2013 Patong Beach, Phuket (Thailand), pp.1-5. http://dx.doi.org/10.15242/IIE.E1213009 